\title{
The Spin-Resolved He II Profile of RX J0558+53
}

\author{
E. T. Harlaftis and K. Horne \\ School of Physics \& Astronomy, University of St. Andrews, North \\ Haugh, St. Andrews, Fife KY16 9SS, Scotland, United Kingdom
}

\begin{abstract}
We observed the new intermediate polar RX J0558+53 with the $4.2 \mathrm{~m}$ WHT in March 1995. We detect 2-3\% pulsations of the He II emission line on the $545 \mathrm{~s}$ spin period. The spin-resolved HeII spectrogram shows a complex structure with two roughly anti-phased components moving from red to blue (double pulse). This is most likely produced by non-axisymmetric gas flow in the rotating magnetosphere of the white dwarf.
\end{abstract}

\section{Introduction}

Intermediate polars form a class of cataclysmic variables and consist of a whitedwarf 'pulsar' and a red dwarf. Mass transfer from the red dwarf forms an accretion disc which is disrupted by the magnetic field of the white dwarf. Gas from the inner-edge of the disc is then funnelled along the magnetic field lines onto the poles of the white dwarf. RX J0558+53 was discovered during the ROSAT all-sky survey and it was optically identified by Haberl et al. (1994) as a $\mathrm{V}=14.6 \mathrm{mag}$ intermediate polar with an orbital period of 4.15 hours. Recently, Allan et al. (1996) identified the 545s spin period of the white dwarf from modulations at 545 -s and 272 -s. We observed RX J0558+53 for 3 hours with the WHT 4.2m at La Palma on 17 March 1995. We used the ISIS spectrograph with TEK CCD chips to cover $6360-6770 \mathrm{~A}$ and $4100-4900 \mathrm{~A}$ at $0.41 \mathrm{~A} / \mathrm{pix}$. The observations were designed so that we sample sufficiently the $545 \mathrm{~s}$ spin period with 6 spectra every $272 \mathrm{~s}$ (total of $2 \times 251$ spectra). The orbital variation of He II 4686 profile is complex. It shows a double-peaked component and an ' $\mathrm{S}$-wave' component crossing from red to blue, indicating a location on the secondary star or the bright spot. The full analysis is in preparation (Harlaftis and Horne, MNRAS, 1997).

\section{Discussion}

Here, we present the spin-resolved spectra of He II 4686 which is the line expected to form through photoionization and therefore the easiest to be related to illumination of gas by a rotating beam from the white dwarf. Using the spin ephemeris given by Allan et al. (1996), we fold all 251 spectra into 12 spin-phase bins, then subtract the average profile to reveal the faint $(2-3 \%)$ spin-period variations. The continuum pulse maxima per spin cycle (( $5 \%$ of steady emission), occur when narrow $\left(200 \mathrm{~km} \mathrm{~s}^{-1}\right)$ Hell components are close their maximum 


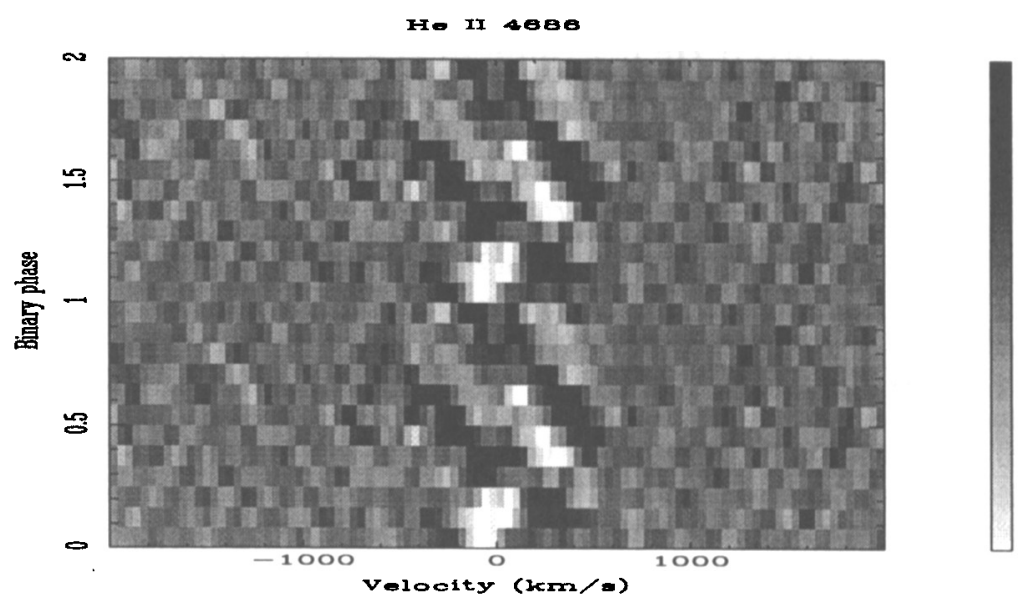

Figure 1. The spin-pulse profile of HeII 4686.

velocity shift, especially at spin phase 0.0 . The first HeIl pulse traverses velocities between +400 to $-500 \mathrm{~km} \mathrm{~s}^{-1}$ during spin phases $0.15-0.65$ and the second pulse moves from about +500 to $-400 \mathrm{~km} \mathrm{~s}^{-1}$ during spin phases $0.45-1.05$. The two He II pulses are simultaneously visible at the continuum pulse maxima. At spin phase 0.5, where the continuum is stronger than at phase 0.0 (Allan et al. 1996), both He II pulses are seen (for phases $0.4-0.8$ ) for a longer period than at spin phase 0.0 . The accretion-curtain model seems plausible to interpret the double-pulse of He II at this early stage of analysis (see, for example, Hellier et al. 1991). Each continuum pulse maximum stands for accretion onto one pole of the white dwarf (Allan et al. 1996). Each He II pulse then may trace the radial flow of gas onto each pole. The first He II pulse is seen at maximum blueshift during the first continuum pulse maximum as the radial flow of gas is accreted onto the receding pole (pole pointing away from observer). The second He II pulse will be seen at maximum redshift as the radial flow of gas is accreted onto the approaching pole (pole pointing towards the observer). GK Per is a system with a 351s spin-period which shows though a single spin pulse in both the continuum and the emission lines (Reinsch 1994). The system's inclination, the angle between the orbital and magnetic axes and asymmetries in the accretion flow, like in the azimuthal extent or differences in the optical depth as viewed from different angles can be combined to model the above observed He II pulses.

\section{References}

A. Allan, et al., 1996, MNRAS, 279, 1345

F. Haberl, et al., 1994, AA, 291, 171

C. Hellier et al. 1991, MNRAS, 248, 233

K. Reinsch, 1994, AA, 281, 108 